\title{
Critical current density of the superconductor $\mathrm{YBa}_{1.5} \mathrm{Ca}_{0.5} \mathrm{Cu}_{3} \mathrm{O}_{7-\delta}$
}

\author{
R B JOTANIA, S M JOSHI, G J BALDHA, H H JOSHI and \\ R G KULKARNI \\ Department of Physics, Saurashtra University, Rajkot 360005, India
}

\begin{abstract}
The real and imaginary (lossy) components of a.c. susceptibility of high- $T_{\mathfrak{c}}$ superconductor $\mathrm{YBa}_{1.5} \mathrm{Ca}_{0.5} \mathrm{Cu}_{3} \mathrm{O}_{7-\delta}$ were measured as a function of temperature for different a.c. fields and frequencies. From the lossy component of the a.c. susceptibility the transport critical current density and its temperature dependence have been computed on the basis of Kim's critical state model.
\end{abstract}

Keywords. Superconductors; critical currents; a.c. susceptibility.

\section{Introduction}

In general in the high- $T_{c}$ granular bulk superconductors it is necessary to distinguish between the superconducting properties of not connected "grains" and of a "bulk" of grains connected through Josephson junctions. A.C. susceptibility measurements as a function of temperature allow one to observe this double behaviour. It is commonly accepted that the low transport critical current density $J_{c}(T)$ in the sintered high- $T_{c}$ superconductors is due to Josephson junction at the grain boundaries (Ekin et al 1987), or, on a small scale, inside the grains (Deutscher and Muller 1987).

No attempt was made to substitute $\mathrm{Ca}$ for $\mathrm{Ba}$ in $\mathrm{Y}-\mathrm{Ba}-\mathrm{Cu}-\mathrm{O}$ system except in our recent work (Baldha et al 1989) of superconductivity in the $\mathrm{YBa}_{2-x} \mathrm{Ca}_{x} \mathrm{Cu}_{3} \mathrm{O}_{7-\delta}$ by resistivity measurements. We report in this paper the determination of critical current density $J_{c}(T)$ by measuring the real $\left(X^{\prime}\right)$ and imaginary $\left(X^{\prime \prime}\right)$ parts of a.c. susceptibility of a sintered $\mathrm{YBa}_{1 \cdot 5} \mathrm{Ca}_{0 \cdot 5} \mathrm{Cu}_{3} \mathrm{O}_{7-\delta}$ as a function of temperature and magnetic field. The method earlier developed by Chen et al (1989) to determine $J_{c}(T)$ from a.c. susceptibility data is used here.

\section{Experimental}

Samples of $\mathrm{YBa}_{1.5} \mathrm{Ca}_{0.5} \mathrm{Cu}_{3} \mathrm{O}_{7-\delta}$ were prepared from high-purity powders of $\mathrm{Y}_{2} \mathrm{O}_{3}$, $\mathrm{BaCO}_{3}, \mathrm{CuO}$ and $\mathrm{CaO}$ mixed in appropriate proportions and pressed into pellets of diameter $1.0 \mathrm{~cm}$ and thickness $0.2 \mathrm{~cm}$. A special technique of sample preparation known as rapid thermal processing (RTP) treatment which will be described elsewhere was used in our work. All samples were synthesized from RTP method at $950^{\circ} \mathrm{C}$ in air. $\mathrm{X}$-ray diffraction shows that the sample consists of a single perovskite-type phase. To study the diamagnetic properties of $\mathrm{YBa}_{1.5} \mathrm{Ca}_{0.5} \mathrm{Cu}_{3} \mathrm{O}_{7-8}$, the real and imaginary (lossy) components of the a.c. susceptibility of this sample were measured as functions of temperature $(4.2 \mathrm{~K}$ to $120 \mathrm{~K})$ for three different values of a.c. field amplitudes $\left(H_{m}\right.$ $=24,240$ and $2400 \mathrm{~A} / \mathrm{m}$ and frequencies $f=10,100$ and $1000 \mathrm{~Hz}$ ). 


\section{Results and discussion}

XRD pattern of $\mathrm{YBa}_{1.5} \mathrm{Ca}_{0.5} \mathrm{Cu}_{3} \mathrm{O}_{7-\delta}$ reveals that it is isostructural with the superconducting $\mathrm{YBa}_{2} \mathrm{Cu}_{3} \mathrm{O}_{7-\delta}$ and it crystallizes in the orthorhombic Pmmm space group with lattice parameters: $a=3.7621(6) \AA, b=3.8942(6) \AA$, and $c=11.697(2) \AA$.

To explain the a.c. susceptibility of high- $T_{c}$ superconductors in the low-temperature region $\left(T<T_{c}\right)$, we use a critical-state model, in which high- $T_{c}$ superconductors are considered to consist of superconducting grains with high $H_{c 1}$ (lower critical field) embedded in a normal or poorly superconducting matrix or network. For simplicity we refer only to matrix hereafter. The matrix allows super currents with a field-dependent critical current density to flow. The complex susceptibility of the sample may be written as

$$
X=f_{g} X_{g}+\left(1-f_{g}\right) X_{m}
$$

where $X_{g}$ and $X_{m}$ are the complex susceptibilities of the assembly of the grains and the matrix, respectively, and $f_{g}$ the volume fraction of the grains.

Normally, the a.c. field amplitude $H_{m}$ is usually lower than $H_{\mathrm{c} 1}$ of grains for temperatures not too close to the $T_{c}$ of the grains, so

$$
X_{g}=-1
$$

Using (2) in (1), we obtain expressions for real and imaginary susceptibility as,

$$
\begin{aligned}
& X^{\prime}=-f_{g}+\left(1-f_{g}\right) X_{m}^{\prime} \\
& X^{\prime \prime}=\left(1-f_{g}\right) X_{m}^{\prime \prime} .
\end{aligned}
$$

For $f_{g}=1, X^{\prime}=-1$ and $X^{\prime \prime}=0$.

The temperature dependence of the real $X^{\prime}(T)$ and imaginary (lossy) $X^{\prime \prime}(T)$ components of the a.c. susceptibility for the superconducting sample $\mathrm{YBa}_{1.5} \mathrm{Ca}_{0.5} \mathrm{Cu}_{3} \mathrm{O}_{7-\delta}$, is shown in figure 1 . As observed from $X^{\prime}$ data in figure 1 , the onset of superconducting transition starts at $82 \mathrm{~K}$. At the lowest applied field (0.3 Oe or $\left.H_{m}=24 \mathrm{~A} / \mathrm{m}\right)$ and at low temperatures $(T<60 \mathrm{~K}), X^{\prime}=-1$ represents the complete diamagnetic shielding of the sample by the supercurrent circulating in the sample surface layer and $X^{\prime \prime}=0$ implies the absence of any losses due to trapped flux. With increasing temperature $(T>60 \mathrm{~K}) X^{\prime}$ decreases whereas $X^{\prime \prime}$ shows a peak near $T_{c}$ at $H_{m}$ $=24 \mathrm{~A} / \mathrm{m}$. The peak in $X^{\prime \prime}$ corresponds to the penetration of supercurrents inside the grains. When the a.c. field is increased the transition is broadened, the $X^{\prime \prime}$ peaks shift to lower temperature. With $H_{m}=2400 \mathrm{~A} / \mathrm{m}(30 \mathrm{Oe})$ the shielding is no longer complete $\left(X^{\prime} \neq-1, X^{\prime \prime} \neq 0\right)$ indicating that the bulk is already penetrated by the magnetic field. Thus at higher a.c. fields, $X^{\prime}$ shows two drops and $X^{\prime \prime}$ two peaks. The high temperature drop and the peak have to be attributed to the grains, the others to the bulk.

Generally, the $X^{\prime}$ of most high- $T_{\mathrm{c}}$ superconductors reaches -1 only in a very small field indicating that the $H_{\mathrm{c}}$ of the matrix to be nearly zero. Under this assumption, the complex susceptibility $X_{m}$ of the matrix is only determined by supercurrents circulating in the matrix. It is well known that the transport critical current density in high- $T_{c}$ superconductors is intergranular in nature and field-dependent (Ekin et al 1987). We use Kim's critical state model (Kim et al 1962,1963) to express the field-dependent intergranular critical current density as,

$$
J_{c}\left(H_{i}\right)=\frac{K}{H_{o}+1 H_{i} 1},
$$



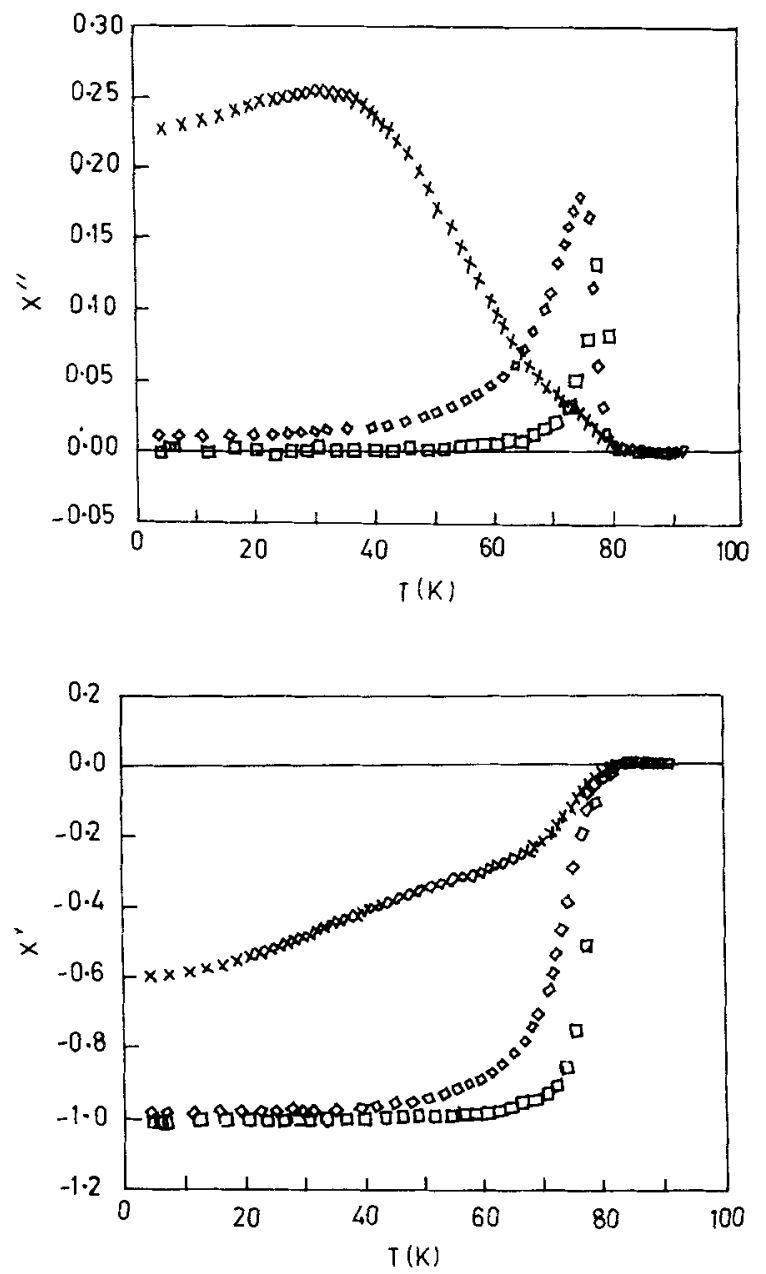

Figure 1. Temperature dependence of the real $\left(X^{\prime}\right)$ and imaginary $\left(X^{\prime \prime}\right)$ components of the a.c. susceptibility at $H_{m}: 24 \mathrm{~A} / \mathrm{m} ; 240 \mathrm{~A} / \mathrm{m} ; X 2400 \mathrm{~A} / \mathrm{m}$.

in which $H_{i}$ is the local internal field and $K$ and $H_{o}$ are positive constants at a given temperature. To obtain $X_{m}^{\prime}$ and $X_{m}^{\prime \prime}$ as functions of a.c. field amplitude $H_{m}$, it is necessary to define a full penetration field $H_{p} . H_{p}$ is the field at which the supercurrent penetrates to the centre of the sample which depends on the shape of the sample. The temperature dependence of intergranular critical current density $J_{c}(T)$ is obtained by measuring $X_{m}^{\prime}$-value and the estimated $f_{g}, X_{m, \max }^{\prime \prime}$ is obtained using (4). Experimentally, one chooses an $H_{m}$ high enough $\left(H_{m} \approx H_{p}\right)$ to obtain $X_{m \text {,max }}^{\prime \prime}$ at a temperature within the defined low-temperature region. We have measured $X^{\prime}(T)$ and $X^{\prime \prime}(T)$ at this $H_{m}=2400 \mathrm{~A} / \mathrm{m}(30 \mathrm{Oe})$ for determining intergranular critical current density $J_{c}(T)$ and its temperature dependence obtained from the lossy component $X^{\prime \prime}(T)$ data at $H_{m}$ $=2400 \mathrm{~A} / \mathrm{m}$. As shown in figure 2 , the computed $J_{c}$ varies almost linearly with $T$ up to $30 \mathrm{~K}$ reaching a value of about $160 \mathrm{~A} / \mathrm{cm}^{2}$ at $30 \mathrm{~K}$ and thereafter it deviates from linearity attaining a value of $185 \mathrm{~A} / \mathrm{cm}^{2}$ at $4 \cdot 2 \mathrm{~K}$. It is found that the field dependence of the transport critical current is not strong in this sample. 


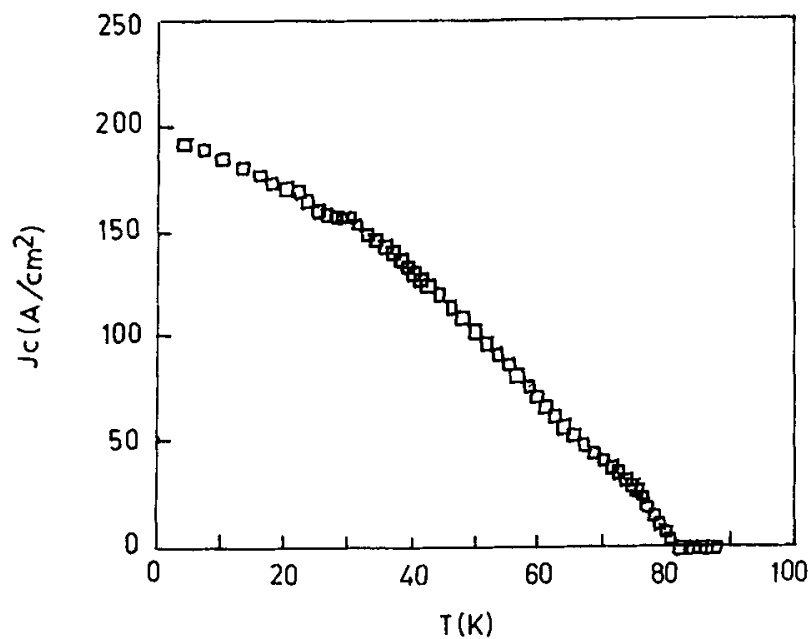

Figure 2. Temperature dependence of the average transport critical current density $J_{c}$ computed from a.c. susceptibility at $H_{m}=2400 \mathrm{~A} / \mathrm{m}$.

\section{Conclusions}

The measured a.c. susceptibility $X^{\prime}$ and $X^{\prime \prime}$ data of sintered $\mathrm{YBa}_{1.5} \mathrm{Ca}_{0.5} \mathrm{Cu}_{3} \mathrm{O}_{7-\delta}$ are separated into two parts: one depending on the grains, the other on the intergranular region. Kim's critical state model has been used to determine the intergranular critical current density from a.c. susceptibility data as a function of temperature.

\section{Acknowledgements}

The authors are thankful to Prof. K V Rao (Sweden) for extending experimental facilities. This work was financially supported by the University Grants Commission, New Delhi.

\section{References}

Baldha G J, Jotania R B, Joshi H H, Pandya H N and Kulkarni R G 1989 Solid State Commun. 71839 Chen D X, Nogues J and Rao K V 1989 Cryogenics 29800 Deutscher G and Muller K A 1987 Phys. Rev. Lett. 5115

Ekin J W et al 1987 J, Appl. Phys. 624821

Kim Y B, Hempstead C F and Strand A R 1962 Phys. Rev. Lett. 9306

Kim Y B, Hempstead C F and Strand A R 1963 Phys. Rev. 129528 